\title{
Cardiac Patients for Non-Cardiac Surgery: Anesthetic Management in Patients with Permanent Pacemaker
}

Eva Oktavia

Anesthesiology Department, Medical Faculty of Ukrida, Jakarta, Indonesia

\section{Correspondence:}

Dr. Eva Oktavia, SpAn, Anesthesiology Department, Medical Faculty of Ukrida, Jakarta, Indonesia .

E-mail: eva.octavia@ukrida.ac.id

\begin{abstract}
Permanent pacemakers (PPM) are often used in patients with electrophysiology disorders. After their device implantation, these patients can undergo cardiac or non-cardiac surgery with general or regional anesthesia. As an anesthesiologist, understanding patients' condition, pacemaker care, and safe anesthetic technique are critical to providing safe patient management. This literature review discussed a systematic approach for managing these patients.

We discussed various approaches and guidelines on anesthesia management of patients with PPM. Particularly, in this review, the American Society of Anesthesiology (ASA) standard was used. Additionally, choices of safe anesthesia techniques should base on patients' clinical condition, the surgical procedure, the duration of surgery, and the convenience of the surgeon.

Overall, patients with PPM require special attention to perioperative management. The anticipation of the patient's condition and the performance of PPM must always be considered to provide safe anesthesia practice.
\end{abstract}

(Indonesian J Cardiol. 2020;4 I:32-39)

Keywords: anesthesia; arrhythmias; electrophysiology; dysrhythmias; pacemaker

\section{Introduction}

$\mathrm{P}$ atients with cardiac diseases are commonly found in anesthesia practice and give a considerable challenge to the anesthesiologists due to higher risks of perioperative morbidity and mortality. The current development and improvement of diagnostic technology enable early detection of electrophysiology disorders in many patients, especially the elderly. In terms of treatment, pacemakers are one of the most reliable documented treatments for both conduction and arrhythmia problems in such patients. ${ }^{1-6}$

More than 3 million patients in the United States have pacemakers and these numbers have been increasing worldwide. In a recent survey from India, Shenthar et al. reported that 37,000 cardiac device implantations take place annually in India and about $80 \%$ of these procedures consisted of bradycardia- 
related permanent pacemaker (PPM) insertions. In Indonesia, the prevalence of bradyarrhythmias with PPM implantations is still unknown but it is undeniably increasing each year. ${ }^{1-5,7}$

As the number of PPM implantation worldwide is getting higher, the number of patients with PPM present for cardiac or non-cardiac surgery also increase. Hence, it is reasonable to expect anesthesiologists to encounter these kinds of patients in their daily practice and to understand the characteristics, problems, and the interaction among PPM device, anesthesia procedure, and anesthesia agents to safely provide anesthesia for patients with any of these implanted devices. ${ }^{1-5,7,8}$

\section{Understanding Permanent Pacemaker (PPM)}

PPM consists of a generator (such as electric circuit and battery) and electrodes accompanied by leads (this will be subsequently referred to as leads), designed for pacing, sensing or both in order to prevent bradycardia. The lithium-iodide batteries are the energy source of pacemaker generator, which can last for 5-10 years and commonly implanted in the infraclavicular region of the anterior chest wall. PPM usually has one or more leads, such as atrial lead and/or ventricular lead, which are implanted using either percutaneous or transvenous method. Leads perform to detect and deliver stimulus to the designated heart chamber based on the device settings. They consist of unipolar (single) and bipolar (dual) leads. For the clinical use, generators with bipolar leads can be programmed into the unipolar mode. ${ }^{2,4,8-14}$

In electrocardiography (ECG), unipolar leads will give the image of spike pacing, because it works to deliver a big electricity vector caused by a large physical gap between leads and generator. Therefore, unipolar leads tend to be easily interrupted because it can pick up extracardiac stimuli and myopotentials, for instance, from thorax joint contraction. Meanwhile, bipolar leads consist of two separate leads and both of them are located in the triggered heart chamber. Both leads are side by side and can result in uninterrupted bipolar trigger. Hence, it is rare to find spike pacing in ECG. ${ }^{4}$, $9-11,13$

Single-chamber pacing has a trigger generator with a lead in the right ventricle. Dual-chamber pacing has a trigger generator with two leads; one is placed on the right atrium while the other one on the right ventricle. There is also biventricular pacing which has a trigger generator with three leads; each of them is put on the right atrium, right ventricle, and coronary sinus in order to make a possible trigger on the left ventricle. Meanwhile, epicardial pacing consists of a trigger generator with leads which is put directly on the surface of the heart (right atrium or right ventricle) through surgery procedure, but this technique is outdated and replaced with a transvenous method. Now epicardial pacing is only used for temporary pacing after openheart surgery and has to be immediately replaced with PPM. In addition, leadless system pacing is a pacing device that consists of a generator and leads put together in one unit and is put on the right ventricle using the transvenous method. ${ }^{2}$

\section{Pacemaker Nomenclature}

Understanding the classification of a cardiac pacemaker can give comprehension regarding the chamber being paced, the chamber being sensed, and the type of response to sensing (as seen in table 1). The five-position system of pacemaker is commonly used based on North American Society of Pacing and Electrophysiology/British Pacing and Electrophysiology Group (NASPE/BPEG) (2002). It is also known as generic codes of the pacemaker. Basic functions of anti-bradycardia are indicated in the first three letters, while the fourth letter explains the program feature

Table 1. Generic codes of pacemaker 4, 9-16

\begin{tabular}{cccccc}
\hline Position & I & II & III & IV & V \\
\hline Category & Pacing & Sensing & Response & Programming & Multisite pacing \\
\hline Letter & $\mathrm{O}$ & $\mathrm{O}$ & $\mathrm{O}$ & $\mathrm{O}$ & O \\
& $\mathrm{A}$ & $\mathrm{A}$ & $\mathrm{T}$ & $\mathrm{R}$ & $\mathrm{A}$ \\
& $\mathrm{V}$ & $\mathrm{V}$ & $\mathrm{I}$ & & $\mathrm{V}$ \\
& $\mathrm{D}(\mathrm{A}+\mathrm{V})$ & $\mathrm{D}(\mathrm{A}+\mathrm{V})$ & $\mathrm{D}(\mathrm{T}+\mathrm{I})$ & & $\mathrm{D}(\mathrm{A}+\mathrm{V})$ \\
\hline
\end{tabular}

A: Atrium, D: Dual, I: Inhibited, O: None, R: Rate modulation, S: Shock, T: Triggered, V: Ventricle. 
which includes cardiac frequency modulation, and the fifth letter describes multisite pacing function. In daily practice, most clinicians use only three-letter model for communication. ${ }^{2,4,8-15}$

A few years ago, pacemaker used fixed rate, but soon it was replaced with a system that could detect spontaneous depolarization of ventricle and prevent unnecessary ventricle stimulus delivery (single chamber atrial/ventricular pacing). $4,10,13,15$

The modern pacemakers can be programmed into various modes. The most common PPM modes are AAI, VVI, and DDD (dual chamber pacing). Pacing with DDD mode can minimize the incidence of pacemaker syndrome and gives more hemodynamic efficiency compared to single-chamber pacing mode. However, the more physiologic PPM mode is DDDR (rate adaptive), because heart activity can be adjusted with patients' metabolic needs during physical, mental, or emotional activity. Several modern PPMs can also automatically shift to other pacing modes based on patients' conditions. ${ }^{4,10,11,13,15}$

\section{Anesthesia Management}

Anesthesia management for patients with PPM can be complicated. Several perioperative guides have been developed for managing these patients. In this review, the American Society of Anesthesiology (ASA) standard was chosen as a framework for clinical approach in perioperative management patients with PPM (as seen in table 2).

\section{Preoperative preparation}

Evaluation and optimization of co-existing diseases preoperatively are necessary. One of the most common co-existing diseases in patients with electrophysiology disorders is heart failure, which is related to the dysfunction of SA and/or AV node to trigger the systolic contraction of the right ventricle and left atrium.4, 9-13

A pre-anesthetic assessment of current PPM type and function is necessary. This can be done by tracking down patients' medical history, medical records, PPM information card, and cardiologist contact number as well as PPM producer. It is also recommended that the PPM function has already been examined at least once in the past twelve months prior to surgery. ${ }^{2-5,8-14,16}$

Another method to determine which PPM type is used by the patients prior to anesthesia is by conducting several routine diagnostic examinations. The necessary minimum examinations are as follows:

- 12 leads ECG: This aims to determine patients' baseline heart rhythm, pacemaker activity, and cardiac pacing.

- Chest x-ray: This is performed to check the PPM position, type, and integration (leads and generator) as well as signs of heart failure.

- Electrolytes: A normal limit should be ensured

Table 2. Perioperative approach for patients with PPM $4,10,13,16$

\begin{tabular}{|c|c|}
\hline Perioperative period & Patients with PPM approach \\
\hline \multirow[t]{7}{*}{ Preoperative } & Indications of implantation \\
\hline & Type and product \\
\hline & Current function mode setting \\
\hline & Can it function well? \\
\hline & How far patients depend on the pacemaker? \\
\hline & Is it necessary to have reprogramming before surgery? \\
\hline & Does anesthesia or surgery affect the performance of pacemaker or vice versa? \\
\hline \multirow[t]{3}{*}{ Intraoperative } & Monitoring \\
\hline & Electrocautery \\
\hline & Defibrillation/emergency cardioversion \\
\hline \multirow[t]{2}{*}{ Postoperative } & Soon after the surgery \\
\hline & Evaluation \\
\hline
\end{tabular}

ASA: American Society of Anesthesiologist, AV: atrioventricular, ECG: electrocardiography, EMI: electromagnetic interference, NIBP: noninvasive blood pressure, PPM: Permanent pacemaker 
because changes in $\mathrm{K}+$ plasma concentration can cause the decrease of heart response towards PPM or defibrillator.

The other diagnostic examinations can be done based on clinical indication. . $^{2,}, 8-10,12-14,16$

The next step is to determine patients' dependency on his/her pacemaker. If it is not possible to confirm with the respective cardiologists or PPM producer, then patients' medical history and preoperative ECG examination can help anesthesiologists determine the perioperative status of patients. History of bradyarrhythmia or syncope which underlies the PPM insertion, or history of $\mathrm{AV}$ node ablation can provide information about patients' dependency on his/her pacemaker. If a spike wave exists at every beginning of $\mathrm{P}$ wave or QRS complex in ECG examination, it can be concluded that the patients' dependency on his/her pacemaker is high. ${ }^{2,4,5,13,16}$

After the evaluation of patients' dependency on his/her PPM, a determination of the implanted PPM reprogramming/resetting should be considered. This is done based on patients' baseline heart rhythm, his/ her hemodynamic status, the upcoming surgical procedure, and the possibility of intraoperative EMI exposure. Patients who are less dependent on his/her PPM do not need to have his/her PPM reprogrammed. Anesthesiologists need to pay attention to fasciculation, shivering, and hyperventilation during the induction or emergence period because they can disturb the function of cardiac frequency modulation in PPM, especially the rate-adaptive type. Stimulation in this respective type of PPM which is due to the aforementioned clinical conditions will be interpreted by PPM as an increase in metabolic activity and can trigger pacemaker tachycardia. ${ }^{2,4,5,9-14,16}$

\section{Intraoperative}

It is necessary to ensure the possibility of EMI exposure during surgery. Electromagnetic exposure towards PPM can change the variety of the pacing setting, depending on the implanted PPM and the specific device producer. The possible effects can include setting alteration, reprogramming, and device deactivation. Therefore, before the surgery, it is crucial to recognize the initial effect which is possible to occur if the applied PPM receives electromagnetic exposure, and the most credible method is by contacting the device producer directly. 2, 4, 5, 9-13

EMI source is derived from electrocautery or diathermy in the operating room. Electrocautery can cause inhibition and/or triggering of asynchronous of an electrical impulse towards cardiac performance. Most surgeries use unipolar electrocautery, but for the surgery of patients with PPM, it is highly recommended to use bipolar electrocautery. Studies report that unipolar electrocautery is far more susceptible to cause PPM interference compared to bipolar coagulation cautery. In bipolar cautery, the electrical current flow is localized between the two poles of the instrument. Meanwhile, in unipolar electrocautery, the electrical current flow is not restricted. Instead, it spreads throughout the body which will increase the risk of electrocautery current flow to intersect the pacemaker device and to trigger device malfunction. Basically, the EMI generated by electrocautery is related to distance and orientation of the current to the patient's PPM device. Therefore, the electrocautery pad must always be located as far as possible from the implanted pacing generator position. During the procedure, anesthesiologists must always be cautious about the risk of intraoperative tachycardia despite the usage of bipolar electrocautery. A defibrillator should be prepared and be available in the operating room prior to surgery. In case malignant tachycardia occurs, defibrillation can be done using the temporary defibrillator. ${ }^{2,4,8-10,13,14,16}$

The usage of shortwave diathermy near the PPM generator can cause an increase in the temperature of the surrounding tissues. Hence, overheating in the generator can happen, which results in the damage of leads insulator. Therefore, the use of intraoperative diathermy should be kept as minimum as, as brief as, and as low as possible. In case of the occurrence of a hemodynamic problem in patients, diathermy usage should be stopped to reduce interruption towards hemodynamic monitoring. In addition, the other sources of EMI that can be found in the operating room are internal and external temporary pacing, transcutaneous nerve stimulator device, and evoked potential monitoring device. Those devices can compete with PPM in performing its function. 2, 4, 5, 8-10, 13, 14, 16

Another thing that should be taken into consideration is the usage of a harmonic scalpel. Even though it does not trigger EMI, it can cut PPM leads. In addition, the harmonic scalpel uses ultrasonic waves, 
causing mechanical dysfunction of PPM, which will trigger ventricular extrasystole (VES). Therefore, it is recommended to put some distance between this device and leads generator, which is at least 15 inches, and to increase the amount of ultrasonic wave gradually based on the expected outcome. ${ }^{4,8-10,13,14,16-21}$

In terms of anesthesia, the induction techniques and anesthetic maintenance depend on the patients' clinical condition. Generally, anesthetic agents do not affect the function of PPM, but it will affect patients' physiologic condition. Therefore, the anesthesia technique need to be adjusted based on the patients' clinical condition., ${ }^{2,4}$ $9,12-14,22$

In general anesthesia, narcotics and inhalational agents can be used safely for patients with PPM. However, for patients with bradycardia, high dosage of fentanyl and dexmedetomidine must be avoided, because it will increase cardiac dependency towards PPM by altering PPM electrical trigger and stimulation threshold. The usage of $\mathrm{N}_{2} \mathrm{O}$ intraoperatively can also cause PPM malfunction due to its infiltration in the possible space around PPM implantation in the pectoral area. This infiltration will cause the accidental removal of anode lead from pacing generator. ${ }^{2,4,9,12-14,22}$

The other agents which should also be avoided for patients with PPM are succinylcholine, etomidate, and ketamine, because they can trigger fasciculation or myoclonus which can interrupt the function of PPM. Succinylcholine can still be given for rapid sequence induction (RSI) on condition that premedication of nondepolarized muscle relaxation must be performed first in order to achieve de-fasciculation. This complication can be averted by doing PPM reprogramming into asynchronous mode prior to induction. Besides those agents, the usage of methadone, haloperidol, ondansetron as well as high dosage of anesthetic inhalation should be avoided towards patients with prolonged QT interval syndrome, because it can increase the risk of polymorphic ventricular tachycardia. ${ }^{2,4,9,12-14,22}$

Neuraxialanesthesiais thefirst choice for patients with PPM, especially for ones with co-existing cardiovascular diseases, because it has less effects on their physiology and enables fast recovery. Nevertheless, it is important to pay attention to the patients' clinical condition, the estimated duration of surgery, the convenience of the surgeons. Spinal anesthesia can be used by giving a low dosage of hyperbaric local anesthesia so that the side effect of hypotension can be reduced. The low dosage of intrathecal opioid as adjuvant should also be considered to increase the effectiveness of analgesia intraoperatively and postoperatively. ${ }^{18-20}$

Another advantage of neuraxial anesthesia technique is that it needs a smaller number of anesthetic agents, thus can reduce the effect on cardiac depression and the other side effects. The suppression of intraoperative stress hormone release is also better than one in general anesthesia, which results in better myocardial oxygenation. Minimal postoperative pain related to the usage of long-acting opioid as an adjuvant leads to faster recovery. ${ }^{18-20}$

Anesthesiologists need to pay attention to the possibility of device migration related to patient positioning and intraoperative positive pressure ventilation. Therefore, the function and the integration of PPM, must always be evaluated before and after performing those procedures. ${ }^{2,4,9,12-14,22}$

Regarding intraoperative monitoring, ASA standardized monitoring devices must always be available and applied before induction. This includes ECG monitor, pulse oximetry (with plethysmograph), and noninvasive blood pressure (NIBP). The usage of invasive monitoring devices (intra-arterial blood pressure and pulmonary pressure monitoring, body core temperature monitoring) perioperatively can be adjusted based on the indication of surgical procedure as well the patients' clinical condition. $4,5,9,11-14,22$

It is crucial be vigilant the ECG monitor during the surgery because patients with PPM are at risk of undergoing dysrhythmia and being exposed to EMI anytime. PPM should be continuously displayed in the ECG monitor by decreasing or adjusting filter sensitivity in the monitor device. Meanwhile, arterial pressure monitoring parameters (such as pulsation palpation, heart rate auscultation, intra-arterial pressure display or ultrasound peripheral pulse monitoring) and pulse oximeter with plethysmography can be applied to confirm the heart rate and the hemodynamic stability. ${ }^{2}$, $4,5,9,11-16,22$

All in all, good monitoring, emergency medicine, temporary pacemaker, and external defibrillator must always be available in the operating room. External defibrillator pads must also be applied before anesthetic induction, which is on the heart apex (left side of anteroposterior breast region) and on axillae, or under 
right clavicle. The distance between defibrillator pads and implanted PPM generator is at least $10 \mathrm{~cm}$ to avoid the possibility of damage in the device as well as device reprogramming. Isoprenaline infusion can be provided in case the increase or improvement of heart rhythm or frequency is needed. $2,4,5,9,10,12,13,15,16,22$

Perioperative PPM failure can be affected by three factors: (1) the failure of pacing generator (this rarely happens), (2) the failure of leads (this also rarely happens. If it does, this is due to the dislocation when positioning patients), and (3) the failure in receiving heart pacing or the increase of pacing threshold (this is more common to happen due to ischemia or myocardial infarction, acid-base and electrolyte imbalances, or high concentration of antiarrhythmic drugs in the circulating blood). If this failure affects the stability of patients' hemodynamic, the following steps can be performed.

1. Give magnetic exposure to PPM. This can be done if it has been previously diagnosed that PPM performance is converted into an asynchronous mode when exposed to magnets. This action will increase the device sensitivity in receiving the trigger of heart pacing.

2. Use a temporary pacemaker. This can be done via transcutaneous, transvenous, and transesophageal.

3. Give pharmacologic therapy to increase heart frequency, the limit of cardiac depolarization, or even both. The agents used are epinephrine (0.5$1 \mu \mathrm{g} /$ minute), dopamine $(5-20 \mu \mathrm{g} / \mathrm{kg} /$ minute), isoproterenol $(0.5-10 \mu \mathrm{g} / \mathrm{kg} /$ minute), or atropine.

4. Find and treat the cause of myocardial ischemia. Ischemia increases energy consumption in order to perform ventricular contraction.

5. Find and treat the possibility of electrolyte imbalance, acid-base disturbance, or side effects of antiarrhythmic drugs.

6. Consider putting an epicardial pacing device surgically if the aforementioned steps do not give a positive outcome. ${ }^{4,11,13,14,22}$

It is important to remember that tachycardia will not be found intraoperatively during hemodynamic monitoring towards patients with PPM especially the ones with a high dependency on his/her pacing device (programmed with fixed-rate), even in hypovolemic condition. Therefore, strict monitoring of intraoperative bleeding and fluid replacement is crucial. ${ }^{2}$
During an intraoperative emergency such as arrhythmia or bradycardia, any magnets should be kept away prior to defibrillation, so that the tachyarrhythmia function of PPM can be reactivated. All possible sources of EMI exposure should be stopped, so the actual heart rhythm and rate can be achieved and diagnosed. If the failure of the PPM function is confirmed, patients will need defibrillation or cardioversion to return their heart rhythm to their initial rhythm. During this period, ECG monitoring must be done continuously in order to determine the ventricular frequency and the heart rhythm, because there is a possibility that the recorded ventricular rhythm is lower than the PPM setting. If that happens, postoperative device reprogramming is needed. However, it is rare to have the device ended in a complete failure despite EMI exposure. The device problems usually consist of inaccurate impulse delivery or excessive increase in pacing frequency (180-200 times/minute). 2

\section{Postoperative}

Patients must always be monitored strictly during the postoperative period and a pacing device with external defibrillator function must always be available until it can be confirmed that PPM is functioning normally. Ideally, the patients' PPM must be under reexamination during this period to check whether its running function or mode is in good condition before sending the patients back to the ward. This is very important especially if problems are encountered during the intraoperative period. ${ }^{2-4,9-16}$

\section{PPM and Central Venous Catheter (CVC) Insertion}

In several non-cardiac operations, such as neurosurgery, spinesurgery, or laparotomy, central venous access needs to be established prior to surgery. This will also be useful for postoperative access in the intensive care unit. Performing CVC insertion in patients with PPM can bring a dilemma to anesthesiologists regarding which insertion site is safer, which safe technique should be used, and what possible complications may occur in such patients. ${ }^{23-25}$

Venous complications after placement of PPM occur in approximately $30-45 \%$ of cases such as stenosis, hematoma, thrombophlebitis, and thrombosis, 
with or without symptoms. The incidence of bacterial infection after transvenous leads placement reported approximately $0.2-4 \%$, meanwhile catheter-related bacteremia after CVC insertion has been estimated 1.65.5 episodes per thousand catheter days. Therefore, it is reasonable to consider that placing an additional CVC within the same path of co-existing transvenous leads may be associated with even greater complications. So far there have not been published guidelines or literature regarding the placement of CVC in patients with implanted pacemaker. ${ }^{23-25}$

Internal jugular and subclavian vein are the most preferable site for CVC insertion. While, in patients with PPM, they have pre-existing implanted cardiac pacemaker in the chest region with pacemaker leads lies in the adjacent venous system. Needle puncture attempt during CVC insertion on the ipsilateral site of PPM should consider the risk of direct contact of CVC guidewire with pacemaker leads intravenously. This condition can trigger leads malfunctioning by triggering inappropriate shock, displacement, or even damaging the implantable cardiac device. Other possible complications are dislodgement of thrombus and disseminated bacterial infection surrounding pacemaker leads, and the possibility of malposition or displacement of central catheter. ${ }^{23,24}$

CVC insertion on the contralateral side of PPM is preferred if no contraindication, such as the presence of tumor or lesions, exists. If ipsilateral side is preferred, it is recommended to consider either the internal jugular or femoral vein as the sites of insertion. ${ }^{23}$

\section{Conclusion}

Patients with PPM and cardiovascular diseases need special attention in perioperative anesthesia management, particularly in terms of patients' clinical condition and PPM performance. Emergency equipment such as a temporary pacing device must always be available in operating and recovery room to treat the possible complication during the perioperative period. It is highly recommended to use intraoperative bipolar electrocautery in the surgery of patients with PPM to avoid PPM interference during surgeries. PPM reevaluation after surgery must be done to ensure that the device works according to the suitable mode and functions properly before patients are being transferred to the ward. When CVC insertion is required, it is recommended to perform the procedure on the contralateral side of the implanted pacemaker to avoid unwanted complications.

\section{References}

1. Morgan G, Butterworth J, Mackey D, Wasnick J. Morgan \& Mikhail's Clinical Anesthesiology Cases. 5th ed. McGraw-Hill Education; 2013.

2. Chakravarthy $M$, Prabhakumar D, George A. Anaesthetic consideration in patients with cardiac implantable electronic devices scheduled for surgery. Indian J Anaesth. 2017; 61(9):736. doi:10.4103/ija. ija_346_17.

3. Govindswamy S, Geetha S. Anesthesia management of an elderly patient having permanent pacemaker for total hip replacement. Karnataka Anaesthesia Journal. 2015; 1(4):194. doi:10.4103/23946954.180655.

4. Rastogi S, Goel S, Tempe D, Virmani S. Anaesthetic Management of Patients with Cardiac Pacemakers and Defibrillators for Non-cardiac Surgery. Annals of Cardiac Anaesthesia. 2005; 8:21-32.

5. Sameera V, Pandia M, Bindu B, Goyal K. Anesthetic considerations and successful management of a patient with permanent pacemaker for cervical spine instrumentation. Saudi J Anaesth. 2018; 12(4):6346. doi:10.4103/sja.SJA_125_18.

6. Bryant HC, Roberts PR, Diprose P. Perioperative management of patients with cardiac implantable electronic devices. BJA Education. 2016; 16(11):388396. doi:10.1093/bjaed/mkw020.

7. Hanafy D, Yuniadi Y, Raharjo S et al. Pedoman Terapi Memakai Alat Elektronik Kardiovaskular Implan (Aleka) Perhimpunan Dokter Spesialis Kardiovaskular Indonesia 2014. Indonesian Journal of Cardiology. 2015:171-245. doi:10.30701/ijc. v35i3.431.

8. Hariharan U, Sonowal S, Kaur M. Pacemaker and Geriatric Anaesthesia: A special report. International Journal of Medical Reviews and Case Reports. 2018;2(Reports in Surgery and Dermatolo):1. doi: $10.5455 /$ ijmrcr.pacemaker-geriatricanaesthesia.

9. Diprose P, Pierce J. Anaesthesia for patients with pacemakers and similar devices. BJA CEPD Reviews. 2001;1(6):166-170. doi:10.1093/bjacepd/1.6.166. 
10. Elsik M, Fynn SP. Permanent pacemakers and implantable defibrillators. In: Mackay JH, Arrowsmith JE, eds. Core Topics in Cardiac Anesthesia. 2nd ed. Cambridge: Cambridge University Press; 2012:241-248. doi:10.1017/ CBO9780511979095.044.

11. Miller R, Cohen N. Miller's Anesthesia. Philadelphia, Pa: Elsevier, Saunders; 2015.

12. Sanghavi P, Shah K, Patel N, Sharma G, Goyal S, Patel B. Perioperative anaesthetic management of patients with cardiac pacemakers in non-cardiac surgery- A Case Report. Gujarat Medical Journal. 2014;69(1):96-97. http://medind.nic.in/gaa/t14/ i1/gaat14i1p96.pdf.

13. Stone M, Salter B, Fischer A. Perioperative management of patients with cardiac implantable electronic devices. Br J Anaesth. 2011;107:i16-i26. doi:10.1093/bja/aer354.

14. Rapsang A, Bhattacharyya P. Pacemakers and implantable cardioverter defibrillators - general and anesthetic considerations. Brazilian Journal of Anesthesiology (English Edition). 2014;64(3):205214. doi:10.1016/j.bjane.2013.02.005.

15. Stoelting R, Hines R, Marschall K. Handbook For Stoelting's Anesthesia And Co-Existing Disease. Philadelphia, PA: Elsevier/Saunders; 2013.

16. Practice Advisory for the Perioperative Management of Patients with Cardiac Implantable Electronic Devices: Pacemakers and Implantable CardioverterDefibrillators. Anesthesiology. 2011;114(2):247261. doi:10.1097/aln.0b013e3181fbe7f6.

17. Cho C, Yu J, Sung L, Chung J, Noh C. Comparison of Percutaneous Nephrolithotomy Using Pneumatic Lithotripsy (Lithoclast ${ }^{\circ}$ ) Alone or in Combination with Ultrasonic Lithotripsy. Korean J Urol. 2010;51(11):783. doi:10.4111/ kju.2010.51.11.783.

18. Smith A. Smith's Textbook Of Endourology. Chichester, West Sussex: Wiley-Blackwell; 2012.

19. Mehrabi S, Zadeh A, Toori M, Mehrabi F. General Versus Spinal Anesthesia in Percutaneous Nephrolithotomy. Urol J. 2013;10(1):756-761. https://www.ncbi.nlm.nih.gov/pubmed/23504678.

20. Movasseghi G, Hassani V, Mohaghegh M et al. Comparison Between Spinal and General Anesthesia in Percutaneous Nephrolithotomy. Anesth Pain Med. 2013;3(3). doi:10.5812/aapm.13871.
21. Canales B, Pugh J. New instrumentation in percutaneous nephrolithotomy. Indian Journal of Urology. 2010;26(3):389. doi:10.4103/09701591.70579.

22. Alex S, Saneesh J, Rao R, Upadya M. Anaesthetic management of a patient with sick sinus syndrome for exploratory laparotomy. Southern African Journal of Anaesthesia and Analgesia. 2010;16(6):24-26. doi :10.1080/22201173.2010.10872713.

23. Sharma P, Singh MK, Vats A, Tobin R, Panigrahi BP. Central venous catheter insertion in patients with transvenous pacemaker or defibrillator leads [letter to the editors]. J Cardiothorac Vasc Anesth. 2011 Aug;25(4):e25. doi: 10.1053/j.jvca.2011.02.018. Epub 2011 Apr 22.

24. Pacana CL, Durand JB. The risk of central venous catheter placement ipsilateral to the permanent pacemaker. JAVA. 2009;14(1):28-30. doi: 10.2309/ java.14-1-5.

25. Bhadauria D, Chellappan A, Gurjar M, Kaul A, Sharma RK, Prasad N. The "dilemma of double lifelines": central venous catheter co-existence with transvenous cardiac pacemaker [letter to the editors]. J Vasc Access. 2017 Jan 18;18(1):e3-e5. doi: $10.5301 /$ jva. 5000622 . 